\title{
Tangence
}

\section{Le protagoniste au centre du réseau informatique : les chemins de l'information dans La piel del tambor ( La peau du tambour ) d'Arturo Pérez-Reverte}

\section{Klaus-Dieter Ertler}

Numéro 55, septembre 1997

La vitesse

URI : https://id.erudit.org/iderudit/025948ar

DOI : https://doi.org/10.7202/025948ar

Aller au sommaire du numéro

Éditeur(s)

Tangence

ISSN

0226-9554 (imprimé)

1710-0305 (numérique)

Découvrir la revue

Citer cet article

Ertler, K.-D. (1997). Le protagoniste au centre du réseau informatique : les

chemins de l'information dans La piel del tambor ( La peau du tambour )

d'Arturo Pérez-Reverte. Tangence, (55), 79-94. https://doi.org/10.7202/025948ar 


\section{Le protagoniste au centre du réseau informatique : les chemins de l'information dans La piel del tambor (La peau du tambour) ${ }^{1}$ d'Arturo Pérez-Reverte}

Klaus-Dieter Ertler

Le Pape et la chaire d'Internet. En seulement 15 jours, depuis que le Vatican s'est branché au service du courrier électronique par Internet, plus d'un million de personnes ont contacté le Pape Jean-Paul II...

L'adresse: http://www.vatican.va

El Pais internacional, 15 janvier1996

Une phrase du journaliste et écrivain français Alphonse Karr ${ }^{2}$ s'est érigée en proverbe, toujours valable dans nos sociétés technologiquement hyperévoluées: "Plus ça change, plus c'est la même chosen. Cette maxime rend comptè des limites inhérentes à la condition humaine et se réfère particulièrement aux constantes mythico-existentielles de l'humanité. Le progrès s'inscrirait, selon ce proverbe, dans une sorte de circularité d'où l'espèce humaine ne sortirait guère.

Mais à y regarder de plus près, il semble reprendre en même temps l'idée du changement et du développement, puisqu'il y fait référence. Depuis la Révolution industrielle du XIX ${ }^{\mathrm{e}}$ siècle en particulier, le progrès technique et technologique dans un monde toujours plus rapide, toujours plus hétérogène, toujours plus informé et maintenant informatisé, a de nombreuses fois déchiré les tissus sociaux - aussi bien au niveau régional que national - pour faire naître de nouvelles constellations, de nouveaux ordres sociaux. Est-ce que ces derniers obtiennent le même statut que les précédents, comme veut nous le faire croire ce proverbe?

1 Arturo Pérez-Reverte, La piel del tambor, Madrid, Alfaguara, 1996, 589 p. Les extraits cités dans l'article ont été traduits par nous.

2 Alphonse Karr, "Les femmes ", Les Guêpes, Paris, Levy, janvier 1849, vol. VI, p. 304 . 
80

Ce n'est pas le lieu ici de résoudre les questions philosophiques soulevées par l'ensemble des développements sociaux depuis deux siècles. Nous nous limiterons plutôt à relever certains changements provoqués par les appareils servant à transmettre des informations, et à relever en particulier les effets de ceux-ci sur les textes littéraires. Dans quelle mesure bouleversentils (ou non) le déroulement d'une fiction, dans quelle mesure touchent-ils l'économie narrative ou les constellations sémiotiques d'un texte?

\section{Prolégomènes à un systémisme fonctionnaliste}

Avant de m'attarder à des questions à la lumière d'un roman européen récent, il est indispensable de proposer quelques balises théoriques afin de comprendre ce qu'on aime à nommer la "société moderne" ou "postmoderne". Pour ce faire, je m'appuierai sur les réflexions de la Théorie des systèmes, élaborée par Niklas Luhmann ${ }^{3}$, un sociologue allemand dont l'approche fonctionnaliste de l'analyse de la société contemporaine fournit une méthodologie intéressante pour l'étude de la littérature, et dont les présupposés ont été discutés par le sociologue français $\mathrm{Michel}$ Maffesoli ${ }^{4}$. La thèse centrale de ses recherches portant sur la société moderne/postmoderne repose sur l'idée suivante: la structure et la fonction de la société actuelle relèveraient d'un haut degré de complexité représentant le développement et la conséquence d'une forte poussée évolutionniste, qui serait marquée par trois formes historiques de différenciation sociale:

a) La différenciation segmentaire comme forme primaire de différenciation, telle que l'ont connue les structures archaïques de notre société. Selon ce principe, la société se compose de différentes structures communautaires d'importance égale, comme le sont, à titre d'exemple, les familles, les tribus ou les sociétés villageoises. Ainsi la structure dans son ensemble demeure relativement simple puisque la communication passe par la (co-)présence de personnes dans une localité bien définie et limitée. La structure n'est pas suffisamment complexe pour aboutir à une intégration

3 Niklas Luhmann, Soziale Systeme. Grundriß einer allgemeinen Theorie, Frankfuit/Main, Suhrkamp, 1984, 675 p.

4 Cf. "Niklas Luhmann", Sociétés. Revue des sciences bumaines et sociales, dir. Michel Maffesoli, Paris, Dunod, 1994, n 43, pp. 3-15. 
de facteurs impossibles, le faible degré de possibilités de variation et de sélection rendant quasi impossible les transformations radicales. Toutes les formes sociales restent ainsi liées aux localisations concrètes, l'actualité passant forcément par la localité.

b) À une échelle supérieure des systèmes de communication se trouve, selon Luhmann, la différenciation stratificatoire, qui s'étend de la transformation des sociétés tribales archaïques aux cultures européennes pré-modernes $\mathrm{du} \mathrm{Xv}^{\mathrm{e}}$ et $\mathrm{XVI}^{\mathrm{e}}$ siècles. La société, après avoir vu éclater ses noyaux sociaux homogènes et fermés (tribaux), s'est formée selon des strates inégales et imperméables, organisée selon une hiérarchie tri- ou quadripartite. Par conséquent, la nouvelle différenciation verticale a connu une forte augmentation de son degré de complexité, dont les codes binaires (haut/bas) ont réglé l'évolution sociale.

c) Selon cette approche, le monde moderne, du $\mathrm{XvII}^{\mathrm{C}}$ siècle jusqu'à aujourd'hui, n'offre plus de divisions à la manière des sociétés archaïques à organisation segmentaire, et il ne recourt pas plus aux hiérarchisations verticales imperméables mais plutôt à la différenciation fonctionnelle de la société. Des systèmes institutionnalisés, rendant chacun compte partiellement, nécessairement, de la société dans laquelle ils évoluent (économie, politique, religion, art, etc.), régis par des codes spécifiques de communication, marquent chacun à sa façon - à un rythme de plus en plus accéléré - le parcours du siècle. Ils rendent compte de la complexité extrême de notre époque, notamment grâce à la multiplicité des points de jonction qu'ils suggèrent avec d'autres systèmes, et des perspectivent qu'ils offrent ${ }^{5}$.

Dans ce contexte, la littérature contribue aux transformations de la société par un système fortement institutionnalisé qui fonctionne - lui aussi - selon un code binaire particulier et un modèle pour observer la société qui lui est propre. En tant que "producteur-remodeleur" de sens, ce système partiel s'intéresse à son environnement social par des procédés spécifiques qui relèvent du discursif, du sémiotique ou du sémantique, en symbiose

5 l.uhman adopte l'expression "schéma de réduction" pour parler du développement des communications modernes. En effet, du téléphone à Internet, l'intégration des réseaux a simplifié les échanges communicationnels. Ccci correspond évidemment en contrepartie à une grande complexification technologique. 
82

avec d'autres modèles dans un cadre systémique clos et avec des paramètres proprement littéraires.

Il est donc tout à fait logique que les nombreux systèmes partiels d'une société organisée selon cette différenciation fonctionnelle, et compte tenu de leurs multiples configurations environnementales selon des focalisations inhérentes à leur systématisation, forgent une réalité extrêmement complexe dont la "réduction constante" (pour reprendre la terminologie luhmannienne) et ultra-rapide de complexité et la création ininterrompue de nouveaux modes et possibilités de jonction est et sera, encore davantage, au cours des prochaines années, d'une première nécessité.

\section{La piel del tambor d'Arturo Pérez-Reverte}

Pour illustrer de quelle façon la littérature contemporaine permet d'observer son environnement social, en particulier les aspects qui concernent la dynamisation des processus communicationnels dans la société moderne, nous avons choisi de nous arrêter au roman espagnol La piel del tambor (La peau du tambour). Le roman, publié à la fin de 1995, n'a pas tardé à connaitre un succès considérable auprès du public hispanophone et se prêtera bien à notre analyse, non seulement par sa thématique, mais aussi par les modifications particulières des procédés narratifs ainsi que par les jeux discursifs mis en texte.

La piel del tambor relève de plusieurs genres romanesques contemporains: roman d'espionnage, d'aventure, de mours, en partie picaresque, en partie hiératique, jouant sur les codes, à la fois subversif et stéréotypé, avec - suivant la "bonne" tradition espagnole - des passages rappelant le récit d'amour, rejoignant le genre idyllique du xIx ${ }^{e}$ siècle, et s'inscrivant, avec les nombreuses références à Carmen, dans le cadre de la tradition purement sévillane.

La narration se construit sur les ruines de la société traditionnelle de l'Europe méridionale. Le nouveau monde est présenté selon une rationalité extrêmement dynamisée des réseaux mondialisés de l'informatique.

Un pirate informatique s'infiltre dans le système central du Vatican et s'enfonce, à la grande surprise des informaticiens jésuites en service de nuit, dans les banques de données, pourtant très 
sophistiquées. Sans encombre, il réussit à franchir tous les obstacles codifiés et tombe sur le code V01A, qui n'est rien de moins que le code de l'ordinateur personnel du Souverain Pontife.

Le message qu'il y dépose ne fait aucune allusion à son identité, mais dévoile une intention nette et bien définie. La lettre adressée au Saint Père "en bonne et due forme" fait référence à la nécessité de se faire entendre par Rome pour un habitant de Séville, ce que les moyens de communication normaux ne permettent pas, compte tenu de la distance entre les deux villes. Seuls des chemins inaccoutumés permettraient de transmettre à Sa Sainteté le fait suivant: une petite église sévillane du $\mathrm{XVII}^{\mathrm{C}}$ siècle se trouve dans le collimateur de marchands et de pouvoirs ecclésiastiques corrompus. Suivent des informations mystérieuses, selon lesquelles "le bâtiment lui-même "serait en train de tuer pour se défendre.

L'identité de Vêpres - c'est ainsi que les jésuites informaticiens ont baptisé l'intrus inconnu - commence à intriguer sérieusement l'administration vaticane, qui fait appel à son service de renseignement spécialisé, le IOE, l'Institut pour les Euvres Extérieures.

Quelques jours plus tard, le père Lorenzo Quart est envoyé à Séville pour découvrir l'identité de Vêpres. Cet agent jure tout à fait avec l'image stéréotypée d'un prêtre, car il s'agit d'un homme mondain et chic qui, par ses manières de s'habiller et de se comporter, rappelle plutôt un cadre ou un militaire qu'un membre du Vatican.

Ce bref résumé du début de La piel del tambor montre bien l'importance des systèmes de communication dans le roman, importance qui n'est pas que thématique mais également formelle. Le lecteur se trouve de prime abord dans un rôle d'observateur. La quête d'un "facteur de communication" par un autre assure la tension du récit, rejoignant en quelque sorte les présupposés - également fonctionnalisés - de la sémiotique structurale. Ainsi naît une recherche, au cours de laquelle le flux des informations sera transmis et/ou traité par les médias.

Au fur et à mesure que le texte progresse, il dévoile les diverses activités auxquelles s'est livré Lorenzo Quart avant sa mission spéciale en Andalousie. À l'occasion d'une conversation de l'agent avec son supérieur, le lecteur apprend que le technocrate réalisait de nombreuses tâches d'espionnage au profit du système 
84

institutionnalisé de l'IOE, surveillant l'orthodoxie dans le monde. Cela l'a mené au Panama de Noriega, puis en Bosnie déchirée par la guerre, ainsi qu'au Brésil, où il relevait les activités conspiratrices auprès des théologiens de la libération. Il représente donc, en quelque sorte, un soldat en costume civil, appartenant à la militia Christi du monde moderne et agissant selon les règles et la tradition de Bernard de Clairvaux.

Une fois arrivé à Séville, il découvre les manigances et les intrigues des décideurs locaux, en particulier celles concernant la petite église du XVII' siècle, Nuestra Señora de las Lágrimas, à laquelle Vêpres fait référence dans sa missive cryptée. La polémique semble tourner autour de la destruction possible de cette église par des gens qui pourraient profiter de la spéculation des terrains, dont les prix ont flambé depuis l'Exposition universelle de 1992.

Le travail de Lorenzo Quart consiste à partir de là à s'infiltrer dans le réseau de communication local afin de prendre connaissance des informations circulant dans celui-ci et d'en dresser un rapport (en espagnol: un informe) à l'intention de l'administration vaticane. Le but étant de fournir le plus vite possible les coordonnées de la personne qui se cache derrière le code de Vêpres. Les rencontres et les conversations qu'il aura au cours de cette mission sont donc nombreuses: à l'occasion de sa première visite de l'église en question, l'agent tombe sur Gris Marsala, perchée sur un échafaudage intérieur en train de photographier les vitraux. Gris, une femme en jeans, la quarantaine, qui s'occupe de la restauration du bâtiment assez délabré, l'accueille avec un accent nord-américain et ne lui ménage pas les compliments sur son physique. Le fait qu'elle soit avertie de la visite d'un messager de Rome prend l'espion au dépourvu.

Contre toute attente, la deuxième rencontre se révèle moins fructueuse: Don Príamo Ferro, le curé simple et rustre de Nuestra Señora, réagit avec agressivité à l'arrivée de cet étranger vêtu de façon urbaine et luxueuse. Sa propre lutte acharnée en faveur du maintien de l'église lui inspire une grande méfiance à l'égard de toute immixtion étrangère, et paradoxalement le dialogue entre les deux hommes ne sera établi que par l'entremise de l'archevêque de la ville, Monseigneur Corvo, bien que celui-ci ne soit pas non plus en bons termes avec l'agent de Rome.

La personne détenant la clé du secret est Macarena Brunner, ultime chaînon d'une illustre lignée sévillane. En elle convergent 
toutes les tensions de la narration. D'un côté, elle est la femme infidèle de Pencho Gavira, le futur directeur de la Banque Chartreuse (Banco Cartujano). Celui-ci planifie par pure ambition et avec tous les moyens possibles à l'appui la destruction de l'église et la vente des terrains. Les rapports mafieux de Gavira avec la pègre de la ville apparaissent en filigrane tout au long du roman, pendant que Lorenzo Quart cherche Vêpres. De l'autre, elle est la descendante du fondateur de l'église et lutte avec le curé Príamo Ferro pour le sauvetage du lieu sacré. Macarena Brunner partage son domicile avec sa mère septuagénaire, aristocrate et distinguée, Cruz Brunner de Lebrija, duchesse du Nouvel Extrême. Lorenzo Quart sera mis dans le secret par un simple geste de miséricorde de la part de Macarena, à laquelle l'unira un lien éphémère de sensualité, avant qu'il ne retourne à Rome et ne soit muté en Amérique latine:

Jamais personne ne devra le savoir, chuchota [Macarena]. Ensuite, elle se mit un doigt sur les lèvres, ouvrit la porte en silence, et les notes de La flute enchantée arrivèrent jusqu'à eux. [...] Là, derrière une porte vitrée à coulisse, complètement ouverte, la lumière d'une lampe de bureau éclairait une table avec un équipement PC sophistiqué, deux moniteurs Sony, haut de gamme, avec imprimante laser et le branchement à une ligne de téléphone. Et assise devant l'ordinateur, avec l'éventail de Romero de Torres et deux bouteilles vides de Coca-Cola, placées sur une pile d'exemplaires du magazine Wired, attentive à l'écran où clignotaient des lettres et des icônes, absorbée dans l'évasion qui la libérait chaque nuit de cette maison, de Séville, d'elle-même et de son passé, voilà Vêpres len la personne de Cruz Brunner de Lebrija], qui naviguait silencieusement à travers le cyber-espace infini. (p. 575)

Dans le déroulement de l'histoire mettant en valeur la quête d'une instance codifiée, un deuxième courant d'information échappe entièrement au messager de Rome: c'est le groupe de contre-espionnage et de sbires, mis en service par le banquier Pencho Gavira à des fins personnelles. Ce réseau, dont les activités se déroulent parallèlement à celles de Lorenzo Quart, et dont l'histoire est intercalée dans la trame narrative principale, permet d'observer les activités de l'agent romain dans une nouvelle perspective. Comparés au réseau fonctionnalisé du Vatican auquel participe son représentant à Séville, les trois personnages de la "zone" sévillane, Potro del Mantelete, Niña Puñales et don Ibrahim avec leur chef, le caïd Celestino Peregil, gardent un contact 
86

avec la rue en y traînant quotidiennement, s'inscrivant dans une conception assez traditionnelle du fonctionnement social, marquée généralement par la lenteur du quotidien.

Dans cette trame parallèle, le code de communication est fonctionnalisé et dynamisé dans la mesure où il se réfère au système économique. Seul l'axe binaire entre "payer" et "ne pas payer "accélère les événements communicationnels entre les quatre personnages et leur destinateur, Pencho Gavira. Celui-ci, qui brigue le poste de directeur de la Banque, fonction emblématique du système économique, voit, rationalise et parle pour tirer profit du maximum de situations, dont la vente des terrains ecclésiastiques de Nuestra Señora. Son garde du corps et assistant Celestino Peregil, l'ancien détective obscur, spécialisé en adultères de bas étage ainsi qu'en fraudes à la Sécurité sociale, rêve de fortune et d'une existence dont les valeurs sont diffusées tous les jours par les médias:

les bars en vogue avec de la musique d'ambiance, le whisky avec beaucoup de glaçons, entrer et sortir des bureaux à la moquette d'une paume d'épaisseur, et le Financial Times sur la table du vestibule, le bourdonnement du fax, l'air climatisé, les secrétaires trilingues. Qu'on soit à Zurich, à New York, qu'on soit à la Bourse de Tokyo, vivre avec des gens qui sentent la lotion, le visage rasé, jouant au golf. C'était formidable de vivre comme dans les publicités à la télé. (p. 56)

Vêpres ne ménage pas les manigances malhonnêtes du banquier ambitieux, qui parfois s'acoquine sans vergogne avec des espions de bas étage. À plusieurs reprises, le pirate informatique s'installe dans le système bancaire de la Cartujano pour harceler Pencho Gavira avec des observations désobligeantes, mais bien fondées.

Les deux trames narratives se rejoignent vers la fin (p. 530), lorsque Lorenzo Quart, Macarena Brunner et son mari attaquent le poste de vigilance des truands qui détiennent en otage - en vue d'une rançon substantielle - le père Príamo Ferro.

\section{Communication accélérée et différenciation fonctionnelle dans le processus narratif}

Après avoir brièvement résumé le récit, il sera opportun de voir dans quelle mesure le texte littéraire est touché au plan 
narratif par les systèmes de communication rapide et la différenciation fonctionnelle dont ceux-ci sont l'expression privilégiée. Suivant les théories de la narratologie traditionnelle, celles de G. Genette ${ }^{6}$, E. Lämmert ${ }^{7}$ et F.K. Stanzel ${ }^{8}$, les questions de focalisation et de perspective, les questions concernant celui qui voit, qui sait et qui parle à l'intérieur d'un récit, ont joué un rôle primordial dans l'analyse littéraire des dernières décennies. Lorsque les médias et les machines à communiquer tels que le téléphone (et plus particulièrement le cellulaire), le télécopieur, le notebook ou Internet interviennent dans le processus de communication des personnages ou du narrateur, le paradigme classique de la narratologie doit être repensé.

Reprenons, à titre d'exemple, le cas du principal protagoniste du roman La piel del tambor, Lorenzo Quart: tout ce qu'il voit, entend, sait et dit s'inscrit à l'intérieur de paramètres au fond très simples et très clairs, qui relèvent des fonctions précises qui lui sont attribuées. Lui, l'agent du service secret du Vatican, s'occupe exclusivement de la quête d'informations qui pourraient lui révéler l'identité du pirate informatique. Sa fonction (dont les codes sont clairs) est de rédiger des rapports (des informations recueillies) sur un ordinateur portatif pour les transmettre aussitôt par télécopieur à Rome. Sa présence soudaine et intempestive au sein de ce système de communication dense, qui se tisse autour du problème de l'église sévillane, modifie, accélère et bouleverse les communications mises en route, à tel point qu'une solution, dans le cas du sauvetage de l'église des griffes des marchands, paraît de plus en plus envisageable.

L'augmentation vertigineuse du flux de l'information et sa transmission accélérée par les médias et les machines à communication transforme le mode d'appréhension du savoir des personnages ainsi que leur perception du réel. En beaucoup moins de temps, ce personnage en voit plus, en entend plus, en sait plus que le protagoniste romanesque traditionnel. L'acte de sa représentation se réalise sous une aura de complexité, dont le processus de réduction - qu'il vienne de lui, de la part d'autres personnages romanesques, du narrateur ou du lecteur (fictif ou réel) crée la nouvelle donne narrative des textes contemporains.

6 Gérard Genette, Figure III, Paris, Seuil, 1972, 286 p.

7 Eberhait Lämmert, Bauformen des Erzäblens, Stuttgart, Metzler, 1955, 280 p.

8 Ftanz Karl Stanzel, Theorie des Erzäblens, Göttingen, UTB, 1979, 340 p. 
88

Cette question est mise en évidence à de nombreuses reprises. Lorenzo Quart se montre toujours surpris - professionnalisme oblige - d'apprendre l'ampleur des connaissances de son entourage, le flux de l'information lui échappant ou dépassant largement le cadre de son travail. "Il est impossible que vous sachiez cela", dit-il à un moment donné à la restauratrice Gris Marsala, qui lui répond à son tour d'une façon on ne peut plus explicite: "Pourquoi? Il existe une invention qui s'appelle le téléphone. On le décroche et on parle" (p. 184). À une autre occasion, le protagoniste se rend compte de sa situation difficile dans un environnement aussi refermé sur lui-même et où de nombreux discours s'enchevêtrent: "Trop d'informations qui circulent, pensa Quart, irrité. On se raconte tout, les uns les autres, dans cet étrange club, et le seul qui obtenait les choses avec difficulté, c'était lui "(p. 410). Un jour, lorsque l'archevêque le menace de dresser un rapport sur son comportement étrange, Quart pense à l'ancienne prison épiscopale:

... il y a quelques siècles, le père Ferro et peut-être lui-même auraient eu - avec beaucoup de probabilité - l'occasion d'échanger des impressions là en-bas, pendant que Monseigneur Corvo envoyait, par voie ordinaire et lente - sa propre version des faits à Rome. Quart réfléchissait sur les avantages de la modernité et du téléphone... (p. 472)

Dans ce nouveau contexte de communication, le téléphone représente parfois non seulement un média, mais un partenaire à part entière. Souvent, les conversations non-médiatisées entre les personnages se voient interrompues pour laisser libre cours à l'intrus téléphonique. Cela empire avec les appareils cellulaires omniprésents et agressifs, rompant encore de manière plus spectaculaire la conversation "traditionnelle" de personne à personne: "La conversation [entre Lorenzo Quart et Siméon Navajo, le policier] fut deux fois interrompue par le bip-bip d'un téléphone portatif, que le policier sortit chaque fois de son sac mauresque en présentant des excuses à Quart ” (p. 476).

L'univers narratif est entièrement dominé par un narrateur omniscient. Mais bien qu'il témoigne de sa connaissance des personnages principaux, en particulier de Lorenzo Quart, il ne semble pourtant pas pénétrer l'écran complexe et nébuleux des contacts communicationnels établis par les médias. Ainsi reste-t-il en deçà des processus communicationnels et participe-t-il intensément aux dialogues en cours sans pour autant échapper à la 
superficialité des messages transcodés. Le message peut donc être "observé "dans sa phase de codification ou bien de réception, mais le narrateur n'arrive pas plus que le lecteur à pénétrer la complexité de ce processus. Par conséquent, l'instance du narrateur ne capte que les messages codés et accessibles à son système. Il joue en quelque sorte avec le flux rapide des données en s'arrêtant au hasard et de façon contingente, semble-t-il, à quelques-unes d'entre elles pour les mettre en discours.

Le narrateur s'éparpille entre divers événements simultanés, s'introduit subrepticement dans les dialogues et même dans la conscience des personnages qui se voit parasitée par les discours des médias. Cette fonction narrative donne un haut degré d'homogénéité au roman. Le résultat en est un texte lisse et équilibré au niveau diégétique et mimétique, qui semble fonctionner comme un écran de télévision où s'inscrivent des images fortes en couleurs qui se suivent à un rythme accéléré. La vitesse de transmission garantie par les médias offre, du point de vue spatial ainsi que temporel, l'image de la société plurifocalisée de communication: simultanéité des événements, contingence des images, choix ludique des cadrages ou caractère superficiel et virtuel de la construction thématique. Par ce procédé, le narrateur reste, certes, l'instance traitant, recyclant et figeant en parole la turbulence vertigineuse de cette complexité, mais il devient surtout l'écran lisse et superficiel par lequel passe l'observation de la société entièrement médiatisée.

Si l'on se penche sur l'aspect symbolique du texte, la polémique qui existe entre l'ordre ancien, basé sur la différenciation segmentaire ou stratificatoire (et, par conséquent, relevant d'un rythme lent) et le monde dynamisé par sa fonctionnalisation, se reconnaît de plusieurs manières. La constellation de cette symbolique rend compte d'une opposition latente dans le roman: d'un côté, les représentants anachroniques en sont le prêtre de l'église agressée par les marchands, Príamo Ferro, ainsi que la famille aristocratique des Brunner (ils se mettent tous les deux au service de la tradition, bien qu'avec des méthodes différentes); de l'autre côté, les représentants du nouveau rythme se retrouvent chez la majorité des personnages, dont Lorenzo Quart, ainsi que chez le narrateur. Cela peut être repéré, de surcrô̂t, au plan topologique à travers les deux villes, Rome et Séville, dont les cadres, en quelque sorte emblèmes de l'ordre séculier de l'organisation traditionnelle, rejoignent les phénomènes de la bipartition. Elles se 
90

voient, de nos jours, agressées par les systèmes sociaux fonctionnels et se trouvent par conséquent en état de liquéfaction communicationnelle. Suivant la logique du système économique, l'église disparaîtrait en peu de temps pour céder la place aux spéculations financières les plus osées. Un autre vecteur de ce genre de développement est le tourisme international, dont les particules transforment l'ordre symbolique des villes.

Voici ce qu'on dit de Rome: "Un groupe de touristes japonais se montra à la porte des salons [du café romain où se trouvent les pères Quart et Spadal, admirant l'intérieur. À la vue des deux collets, quelques-uns souriaient avec des inclinations courtoises" ( $\mathrm{p}$. 49). Et voici maintenant ce qu'on dit de Séville:

Il y avait des fiacres, des vendeurs de cartes postales, [...] et des touristes qui regardaient vers le haut, étonnés, pendant qu'ils faisaient la queue pour visiter la tour [Giralda]. Une jeune fille étrangère à l'accent nord-américain se détacha du groupe pour poser, à Quart, une quelconque question banale sur une direction proche de la place: un prétexte pour observer de près son teint bronzé, tranquille [...] (p. 113).

La symbolique se référant indirectement à la lenteur ou même à l'impossibilité des communications peut être observée également par la présence directe d'une image dans le texte: à la page 153, le lecteur tombe sur une carte postale de l'église sévillane du siècle dernier mettant en valeur la frustration d'une ancêtre de Macarena Brunner dont la communication a été interrompue; il s'agit d'une des nombreuses missives adressées à l'amant de la jeune femme et interceptées - à l'époque - par le pater familias intraitable.

La symbolique manichéenne se poursuit aussi dans la présentation des images ecclésiastiques. La lenteur ainsi que la vitesse correspondent au modèle vétuste ou hyper-moderne. Lorsque le père Quart dit au curé de Nuestra Señora: "Votre église est la télévision du Xvis ${ }^{\mathrm{e}}$ siècle?" celui-ci répond par une comparaison:

Et alors! [...] Mes vieilles pierres, mon retable et mon latin sont plus dignes que toutes ces chansons avec mégaphonie, les écrans géants et la Sainte Messe convertie en spectacle pour des masses étourdies par l'électronique. (p. 329)

Il suffit de vous regarder : [...] Vous faites partie de ces prédicateurs à la télé, pasteurs d'une église sans âme, qui parlent des fidèles avec la [même] langue qu'emploient les télévisions pour se référer au public. (p. 325) 
Après avoir mis en exergue quelques observations sur les transformations opérées sur les plans narratif et symbolique, nous nous consacrerons brièvement au problème discursif tel qu'il se pose dans le roman de Pérez-Reverte. Malgré les apparences, la tension et l'intérêt du texte résident moins dans sa thématique que dans la subversion et la transformation des discours traditionnels grâce à l'impact des communications réalisées à l'aide des médias. Nous savons que les discours médiatisés selon le principe de la mondialisation et de la simultanéité ${ }^{9}$ transgressent non seulement les frontières de nombreux systèmes culturels, mais transforment aussi les ensembles discursifs traditionnels pour créer de nouveaux espaces virtuels, en privilégiant l'éphémère ${ }^{10}$ et l'actuel.

Dans le roman de Pérez-Reverte, l'effet de subversion ou de changement de paradigme atteint son apogée lorsque le chevauchement de discours se réalise (et peut être observé) par le télescopage de deux discours antagonistes: celui du Vatican, symbole de l'éternel et de l'orthodoxie, et celui de la société fonctionnalisée, privilégiant l'efficacité et les lois du marché, la mondialisation et l'informatisation. La question se pose alors de savoir si le Vatican s'est mis au diapason de la communication moderne en intégrant l'informatique dans son service de communication et en s'accordant aux discours actuels, dont Quart serait le porteparole, ou bien si, au contraire, le monde informatisé a purement et simplement avalé le centre traditionnel de l'Église catholique.

Les deux mouvements semblent se rejoindre au plan discursif, où de nombreuses indications laissent deviner l'un ou l'autre. On se rend compte que le discours religieux se voit intégré dans le tourbillon du discours social, canalisé par les médias de communication qui, grâce à leurs moyens de diffusion, le divulguent à une vitesse accélérée à travers le monde.

La mise à jour des argumentations, des lectures et des anciennes doctrines théologiques forme le point de départ du premier chapitre du livre: le Vatican y est présenté comme une forteresse à défendre contre les intrusions extra-institutionnelles, dont les

9 Cf. Götz Grossklaus, «Neue Medienrealität *, Theo Elm/Hans H. Hiebel (dir.), Medien und Maschinen. Literatur im techniscben Zeitalter, Freiburg, Rombach Verlag, 1991, p. 185.

10 Gilles Lipovetsky, L'empire de l'éphémère, Paris, Gallimard, 1987, $340 \mathrm{p}$. 
agressions seraient à éliminer. Le discours technique des spécialistes jésuites sur les possibilités d'une infiltration informatique au début du roman, permet de voir se dessiner en filigrane la présence du service de reconnaissance (IOE) en tant que corps inquisitorial. Si l'on s'occupe sur le plan informatique de sigles techniques comme INMAVAT, V01A ou HK, si l'on alarme les supérieurs par téléphone pour leur annoncer la présence d'un pirate dans le système, si l'on raisonne sur les "très jeunes garçons qui se sont spécialisés à naviguer à travers les lignes téléphoniques en explorant les systèmes inconnus pour défier le plus difficile", si l'on parle "de junkies du chip, acquis à la haute technologie, qui s'amusent à chercher fortune à la Chase Manhattan Bank, le Pentagone ou le Vatican" (p. 14), on met en route le service spécialisé en affaires d'investigation. Celui-ci se réfère pourtant aux valeurs inquisitoriales de l'église pour sauver la doctrine et argumente avec les présupposés de la Congrégation pour la Doctrine de la Foi, autrement dit le Saint Office. Il s'en tient au latin et distingue bien les termes officiels et officieux, mettant l'accent sur le dernier. "Le terme "officieux" faisait partie du langage du Vatican et avait le caractère spécial de ce qui se pensait par rapport à ce qui se disait; même de ce qu'on a dit, bien qu'on n'admettrait jamais l'avoir dit" (p. 23). Aux yeux de Quart, l'orthodoxie exige surtout deux valeurs des professionnels du service secret: loyauté et prudence. L'archevêque en service à Rome constate même le caractère hypercorrect de l'agent Quart: "Bernard de Clairvaux et ses Templiers mafieux auraient fait bon ménage avec vous. Je suis sûr que - une fois emprisonnés par Saladin - ils se seraient fait couper la gorge en morceaux avant de renier leur foi. Non pas par piété, évidemment. Par orgueil" (p. 44).

Le glissement rapide et ludique d'un type de discours à un autre qui caractérise le roman se fait encore plus sensible dans d'autres champs sémantiques que nous nous contenterons d'énumérer: le champ économique du banquier et de ses espions, le champ du discours amoureux entre l'ancêtre de Macarena et son amant ainsi qu'entre Macarena et Quart, le champ costumbriste et spatial de Rome, mais surtout de Séville, et finalement le champ volatile et fantasque des médias dont le roman rend compte non seulement sur le plan diégétique mais également structural.

Le champ discursif permet donc d'exprimer, en quelque sorte, deux mouvements contradictoires et opposés : d'un côté est 
souligné, par la quête de l'agent de renseignement, le caractère homogène de la structure sémantique/sémiotique qui mène le récit vers la solution du problème posé, l'identification de Vêpres, le pirate informatique; de l'autre, les champs de discours polyfocalisés par les nombreuses voix, en partie attribuables aux différents systèmes de transmission, dynamisent et accélèrent le récit et - selon leur attribution - faisaient éclater celui-ci dans tous les sens comme un hypertexte, le rendant insaisissable au plan axiologique. Les discours flous et glissants, protéiformes et cyniques se voient projetés comme sur un écran: ils semblent évoluer, de façon vertigineuse, dans une espèce de vacuum axiologique.

Cette vitesse dans la succession des images, la kyrielle de personnages, souvent falots, qui défilent dans le roman, les multiples fonctions codifiées, mécanisées par les médias et l'ensemble des machines à communication, tout cela donne une impression de superficialité dont on peut parier qu'elle correspond au désir de l'auteur. Aux yeux de celui-ci, seul un élément semble susceptible d'être sauvé, c'est la ville de Séville. "Car, souligne-t-il dans l'épigraphe, personne ne pourrait inventer une ville comme Séville". Et, à la fin du roman, l'auteur n'hésite pas à ajouter un plan de la ville pour donner un cachet de vérité aux lieux et à l'image du litige : le Nuestra Señora de las Lágrimas.

Ainsi le roman de Pérez-Reverte est une sorte de mise en texte de notre société de communication fonctionnalisée et structurée selon de multiples systèmes automatisés. À plusieurs égards, l'importance des réseaux de transmission moderne de l'information s'impose dans le roman, que ce soit dans la thématique choisie, dans les fonctions narratives, dans les réseaux symboliques ou dans les champs discursifs multiples qu'on y retrouve. À telle enseigne qu'on pourrait se croire devant un texte produit par l'informatique. Les valeurs se trouvent dans un flottement permanent, seul le canevas sémantico-narratif et le cadre urbain semblent, par leur caractère factuel, toujours correspondre à un monde clair ${ }^{11}$ : trouvaille de l'objet recherché par un sujet au service d'un destinateur pourvu d'un ordre précis, favorisé par des instances adjuvantes et harcelé par des opposants dans la recherche du but.

La piel del tambor témoigne du changement effectué dans le paradigme perceptif à notre époque: sur plusieurs plans rend-il 
94

compte de l'accélération du cadre spatio-temporel, et même de la perte de certains aspects de celui-ci. Ce changement de paradigme ne serait pas pensable sans la présence massive des médias aujourd'hui, accélérant le flux communicationnel et passant outre non seulement les barrières installées par l'espace et les cultures mais aussi certains cloisonnements de l'organisation stratificatoire de la société. Ainsi, le roman de Pérez-Reverte peut être vu comme un texte fort caractéristique de notre époque pour avoir rendu compte des fluctuations qui se dissimulent derrière la répartition du monde en systèmes de communication fonctionnalisés. 